\title{
U-duality and M-theory, an algebraic approach
}

\author{
by Niels A. Obers ${ }^{a}$ and Boris Pioline ${ }^{b}$ \\ ${ }^{a}$ Nordita and Niels Bohr Institute, Blegdamsvej 17, DK-2100 Copenhagen, Danmark \\ ${ }^{b}$ Centre de Physique Théorique, École polytechnique, F-91128 Palaiseau, France \\ E-mail : 'obers@nbi.dk', 'pioline@cpht.polytechnique.fr'
}

ABstract: Based on our work [1], we discuss how U-duality arises as an exact symmetry of M-theory from T-duality and 11D diffeomorphism invariance. A set of Weyl generators are shown to realize the Weyl group of $S O(d, d, Z)$ and $E_{d(d)}$, while Borel generators extend these finite groups into the full T- and U-duality groups. We discuss how the BPS states fall into various representations, and obtain duality invariant mass formulae, relevant for the computation of exact string amplitudes. The realization of U-duality symmetry in Matrix gauge theory is also considered.

\section{Introduction}

Once thought of as distinct ten-dimensional consistent perturbative theories, the five superstring theories are now understood as five different asymptotic expansions of a unifying eleven-dimensional M-theory in different corners $g_{s} \rightarrow 0$ of its moduli space, with string dualities providing the transition functions between these patches[izin. Due to the asymptotic nature of string perturbation theory, this still only covers a zero-measure set of the moduli space, while analytic continuation to finite $g_{s}$ can be achieved for quantities that are protected from quantum corrections, such as the BPS spectrum and BPS-saturated amplitudes. One-loop exact amplitudes on one patch translate into non-perturbative amplitudes in the dual one, and world-sheet instantons turn into semiclassical configurations of Euclidean branes wrapped on non-trivial cycles of the target space.

On the other hand, non-perturbative symmetries identify different points in the moduli space and hold for any amplitude. The prototypical example is the $S l(2, Z)$ S-duality symmetry of ten-dimensional type IIB theory, which was used to obtain the exact four-graviton $R^{4}$ amplitude as the weight $s=3 / 2 S l(2, Z)$ Eisenstein series [귄.

$$
E_{s=3 / 2}^{S l(2, Z)}=2 \zeta(3) \sum_{p \wedge q=1} \alpha_{(p, q)}^{3}
$$

where $\alpha_{(p, q)}=\sqrt{\tau_{2}} \alpha^{\prime} /|p+q \tau|$ is the Einsteinframe inverse tension of a $(p, q)$ string, and $\tau=$ $a+i / g_{s}$ the complex modulus in the upper half plane. This result follows from supersymmetry constraints and S-duality invariance $\left[\begin{array}{l}4 \\ 1\end{array}\right]$. The weak coupling expansion reproduces the tree-level and one-loop contributions, implies perturbative nonrenormalization beyond one-loop, and exhibits a sum of D-instanton effects. More generally, exact amplitudes should be given by (non-holomorphic) automorphic forms of the symmetry group, selected on the basis of the leading perturbative behaviour and supersymmetric constraints [5in] . From a study of this and other exact results, one may hope to infer the rules of instanton calculus in

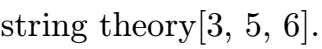

The purpose of this talk is to extend these considerations to the lower-dimensional case of M-theory compactified on a torus $T^{d}$, where the symmetry gets enlarged to the U-duality group $E_{d(d)}(Z)[\bar{i} \overline{1}]$, and correspondingly more states come into play. We first review some basic facts about M-theory and its relation to type IIA string theory, then discuss the continuous hidden symmetry [1] of toroidally compactified 11D supergravity. Only a discrete subgroup of this symmetry may remain as a quantum symmetry of M-theory, and we show how this U-duality follows from Tduality and 11D diffeomorphism invariance. We first focus on a set of Weyl generators of these groups, and derive how the BPS spectrum falls into representations thereof [9in] tors are then included to obtain duality invariant mass formulae. Finally, we use the connec- 
tion between M-theory in Discrete Light-Cone Quantization and Matrix gauge theories [1] $\left.{ }_{1}^{\prime}\right]$ to uncover the spectrum of the latter theories, and discuss the realization of U-duality in these nongravitational theories. This talk is a shortened version of Ref. [1] 1$]$, to which the reader is refered for a more extensive discussion and list of references.

\section{M-theory, BPS spectrum and hid- den symmetries}

M-theory was originally introduced [2] coupling limit of type IIA string theory. It reduces to $11 \mathrm{D}$ supergravity at low energies, and is conjectured to exhibit eleven-dimensional $\mathrm{N}=1$ super Poincaré invariance. In particular, M-theory compactified on a circle $S^{1}$ of radius $R_{s}$ gives type IIA string theory with

$$
R_{s} / l_{p}=g_{s}^{2 / 3}, \quad l_{p}^{3}=g_{s} l_{s}^{3}
$$

where $l_{p}$ denotes the $11 \mathrm{D}$ Planck length, and $g_{s}$ and $l_{s}$ the string coupling and length. Indeed, one observes that at strong coupling $g_{s} \rightarrow \infty$ the eleventh direction decompactifies. The massless spectrum of type IIA string theory gets unified in the $11 \mathrm{D}$ setting as

$$
g_{M N} \simeq\left(g_{\mu \nu}, \phi, \mathcal{A}_{\mu}\right) \quad, \quad \mathcal{C}_{M N R} \simeq\left(B_{\mu \nu}, \mathcal{C}_{\mu \nu \rho}\right)
$$

in terms of the M-theory graviton and antisymmetric three-form and the NSNS fields $(g, B, \phi)$ and $\mathrm{RR}$ gauge potentials $\left(\mathcal{A}_{1}, \mathcal{C}_{3}\right)$ of type IIA string theory (the lower index denotes the number of antisymmetric indices); so do the half-BPS states, charged under these fields:

$$
\begin{aligned}
K K=(D O, K K), & M 2=(F 1, D 2), \\
M 5=(D 4, N S 5), & K K 6=(K K 5, D 6, \ldots)
\end{aligned}
$$

Here the first (resp. second) entry in each parentheses corresponds to longitudinal (resp. transverse) reduction with respect to the eleventh direction. This identification can be most easily seen from the mass or tension of the M-theory BPS states, $\mathcal{T}_{0}=1 / R_{s}, \mathcal{T}_{2}=1 / l_{p}^{3}, \mathcal{T}_{5}=1 / l_{p}^{6}$, $\mathcal{T}_{6}=R_{T N}^{2} / l_{p}^{9}$ for the Kaluza-Klein momentum state, M2 and M5 brane, and KK6-brane (or Taub-NUT monopole), using the relations in (2.1).
As a side remark, the KK6-brane may also be reduced along a non-compact direction of the Taub-NUT space, giving rise to a state with tension proportional to $R_{T N}^{2} R_{s} / l_{p}^{9} \sim 1 / g_{s}^{3}$ and a logarithmically divergent geometry at infinity (first reference in $\left[1 \overline{1} \overline{1}_{1}^{\prime}\right)$. As we shall see, U-duality predicts many more such states with exotic tensions $\mathcal{T} \sim 1 / g_{s}^{p \geq 3}$ for compactification to $D \leq 3$ di-

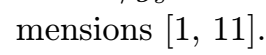

Upon compactification on a torus $T^{d}, 11 \mathrm{D}$ SUGRA exhibits a group of continuous global symmetry $G$, as well as a (composite) gauge invariance under its R-symmetry maximal compact subgroup $K$, as listed in Table 1 [8]i]. The symmetry can be seen among the scalar fields, which take value in the symmetric space $K \backslash G$. The local $K$-invariance can be gauge-fixed thanks to the Iwasawa decomposition

$$
G=K \cdot A \cdot N
$$

into maximal compact $K$, Abelian $A$ and nilpotent $N$ subgroups. A natural gauge is obtained by taking $K=1$, so that the moduli can be represented as a vielbein: $\mathcal{V} \in A \cdot N$ or equivalently as a $K$-invariant matrix $M=\mathcal{V}^{t} \mathcal{V}$. In this representation, the Abelian factor $A$ is parametrized by the radii $R_{I}$ of the internal torus, while the nilpotent factor $N$ incorporates the expectation values of the off-diagonal components of the metric and of the gauge potentials $\mathcal{C}_{3}$ on the torus, as well as their Poincaré-dual $\mathcal{K}_{1 ; 8}$ and $\mathcal{E}_{6}$. The symmetry group $G_{d}$ acts on the right on the coset $K \backslash G$, and induces a compensating moduli-dependent $K$-rotation to preserve the gauge $K=1$. The massless spectrum also includes a number of $p$ forms, given for $p=1,2$ in the last two columns of Table 1, where dualization into forms of lower degree has been carried out. They transform linearly under $G$ and induce charges for particles $(m)$ and strings $(n)$ respectively. Those are linearly related to the central charges in the $N=8$ supersymmetry algebra by $Z=\mathcal{V} \cdot m$, so that the invariant quadratic form

$$
\mathcal{M}^{2}=Z^{t} Z=m^{t} M m \quad, \quad M=\mathcal{V}^{t} \mathcal{V}
$$

is precisely the invariant mass for half-BPS states (quarter-BPS states receive further corrections).

While the low-energy effective action is invariant under the continuous group $G$, the exis- 


\begin{tabular}{|r|r||l|l|l|l|l|}
\hline$D$ & $d$ & $G=E_{d(d)}$ & $K$ & scalars & 1-form & 2 -form \\
\hline 10 & 1 & $R^{+}$ & 1 & 1 & $\mathbf{1}$ & $\mathbf{1}$ \\
9 & 2 & $S l(2, R) \times R^{+}$ & $U(1)$ & $4-1$ & $\mathbf{3}$ & $\mathbf{2}$ \\
8 & 3 & $S l(3, R) \times S l(2, R)$ & $S O(3) \times U(1)$ & $11-4$ & $\mathbf{( 3 , 2 )}$ & $\mathbf{( 3 , 1 )}$ \\
7 & 4 & $S l(5, R)$ & $S O(5)$ & $24-10$ & $\mathbf{1 0}$ & $\mathbf{5}$ \\
6 & 5 & $S O(5,5, R)$ & $S O(5) \times S O(5)$ & $45-20$ & $\mathbf{1 6}$ & $\mathbf{1 0}$ \\
5 & 6 & $E_{6(6)}$ & $U S p(8)$ & $78-36$ & $\mathbf{2 7}$ & $\mathbf{2 7}$ \\
4 & 7 & $E_{7(7)}$ & $S U(8)$ & $133-63$ & $\mathbf{5 6}$ &. \\
3 & 8 & $E_{8(8)}$ & $S O(16)$ & $248-120$ &. &. \\
\hline
\end{tabular}

Table 1: Cremmer-Julia symmetry groups and scalars, 1-forms and 2-forms.

tence of charged states implies that the symmetry remaining at the quantum level is at most a discrete subgroup thereof. In particular, some charges correspond to momenta in compact directions (resp. string or brane wrapping numbers around cycles of the torus), and therefore take values in the reciprocal (resp. homology) lattice. The most convenient constraint takes place in $D=4$, where the quantum symmetry should preserve the symplectic Dirac quantization constraint $[\bar{i} \bar{i} \mid$ :

$$
E_{d(d)}(Z) \subset E_{7(7)}(R) \cap S p(56, Z) .
$$

On the other hand, 11D diffeomorphism invariance implies that the mapping class group $S l(d, Z)$ of the torus $T^{d}$ be an exact symmetry, whereas T-duality holds to all orders in type IIA string perturbation theory and therefore in M-theory as well. These two symmetries certainly preserve Dirac quantization, and the U-duality group of M-theory is therefore

$$
E_{d(d)}(Z)=S l(d, Z) \bowtie S O(d-1, d-1, Z),
$$

where the symbol $\bowtie$ denotes the group generated by the two non-commuting subgroups. In the following, we elucidate this equality by studying the action of a set of Weyl and Borel generators.

\section{Dynkinese approach to T- and U- duality}

We first construct a set of Weyl generators for the T-duality symmetry group $S O(d, d, Z)$ of toroidally compactified type II string theory. The moduli space of the NSNS sector of type II string theory on $T^{d}$ displays a structure analogous to $(2.3)$, with now $G=S O(d, d, R)$ and $H=S O(d) \times$ $S O(d)$, where $A$ are the radii of the torus and $N$ the off-diagonal components of the metric and antisymmetric tensor. There is also an extra factor $R^{+}$for the dilaton. We define the Weyl generators as those preserving a rectangular torus with zero two-form vev, namely $N=1$. A minimal generating set is provided by the exchange of radii and a double $\mathrm{T}$-duality on two directions (a single T-duality would map to type IIB):

$$
\begin{gathered}
S_{i}: R_{i} \leftrightarrow R_{i+1}, \quad i=1 \ldots d-1 \\
T:\left(g_{s}, R_{1}, R_{2}\right) \leftrightarrow\left(\frac{g_{s}}{R_{1} R_{2}}, \frac{1}{R_{2}}, \frac{1}{R_{1}}\right)
\end{gathered}
$$

In order to understand the structure of this group, we represent the tension of a state $\mathcal{T}=$ $g_{s}^{x^{0}} R_{1}^{x^{1}} R_{2}^{x^{2}} \ldots R_{d}^{x^{d}}$ as a vector $\lambda=x^{0} e_{0}+\ldots x^{d} e_{d}$ in a weight space, on which the generators $S_{i}$ and $T$ act linearly. In fact the metric on this space can be chosen so that they are orthogonal reflections

$$
\begin{aligned}
\lambda \rightarrow \rho_{\alpha}(\lambda) & =\lambda-2 \frac{\alpha \cdot \lambda}{\alpha \cdot \alpha} \alpha \\
(\lambda, \lambda) & =-\left(x^{0}\right)^{2}+\left(x^{i}\right)^{2}+x^{0} x^{i}
\end{aligned}
$$

with respect to planes normal to the set of simple roots $\alpha_{0}, \alpha_{i}$. The non-Euclidean metric of the space can be evaded by noting that the $D=$ $(10-d)$-dimensional Planck length $V_{R} / g_{s}^{2} l_{s}^{8}$ is invariant, and restricting to the subspace orthogonal to $\delta=e_{1}+\ldots+e_{d}-2 e_{0}$. The group structure is then determined by the inner products between roots, summarized in the Dynkin diagram

$$
\begin{gathered}
\circ_{0}\left(\frac{1}{g}\right) \\
\mid \\
\left(\frac{R_{1}}{g}\right) \circ_{1}-\circ_{2}-\circ_{3}-\ldots-\circ_{d-1}\left(\frac{1}{R_{d}}\right)
\end{gathered}
$$


which is precisely the one of $S O(d, d)$. On this diagram, we have also indicated the tensions of the fundamental weights associated to the the extremal nodes, which show that the KK modes on the torus together with the string winding states transform as a vector, while the two spinor representations correspond to a multiplet of Dparticles and D-strings respectively.

We now apply the same techniques to the Weyl group of the U-duality group (2.5) of Mtheory on $T^{d}[\overline{9}]$. In this case, we first need to translate the double T-duality (3.2) in M-theory variables using (2.1) , and conjugating this with an 11D diffeomorphism $R_{s} \leftrightarrow R_{i}$, we find the $Z_{2}$ symmetry

$$
T_{I J K}: \quad R_{I} \rightarrow \frac{l_{p}^{3}}{R_{J} R_{K}}, \quad l_{p}^{3} \rightarrow \frac{l_{p}^{6}}{R_{I} R_{J} R_{K}}
$$

which involves a set of three directions. A minimal set of generators of the Weyl group can be chosen as $T=T_{123}$ and the permutations $S_{I}: R_{I} \leftrightarrow R_{I+1}$. The action of the U-duality Weyl group is now represented on a weight vector $\lambda=x^{0} e_{0}+\ldots x^{d} e_{d}$ by considering the tension monomials $\mathcal{T}=l_{p}^{3 x^{0}} R_{1}^{x^{1}} R_{2}^{x^{2}} \ldots R_{d}^{x^{d}}$. The generators $T$ and $S_{I}$ can again be implemented as orthogonal reflections (3.3) for the F-theory-like metric $(\lambda, \lambda)=-\left(x^{0}\right)^{2}+\left(x^{i}\right)^{2}$, with respect to roots $\alpha_{0}, \alpha_{I}$ with inner products

$$
\begin{gathered}
\circ_{0}\left(\frac{1}{l_{p}^{3}}\right) \\
\left.|| \frac{R_{1}}{l_{p}^{3}}\right) \circ_{1}-\circ_{2}-\circ_{3}-\circ_{4}-\ldots-\circ_{d-1}\left(\frac{1}{R_{d}}\right)
\end{gathered}
$$

Not surprisingly, this is the Dynkin diagram of the $E_{d(d)}$ groups. Note that for $d=9$, the invariant Planck length vector $V_{R} / l_{p}^{9}$ becomes null, and it is no longer possible to go to the orthogonal subspace. This is consistent with the fact that $E_{9}$ is the affine Lie group associated to $E_{8}$, and implies that the representations are infinite dimensional. For $d>9$, the symmetry is even more dramatic, with hyperbolic $E_{10}$ or Kac-Moody $E_{11}$, while for $d<9$ we recover the groups in Table 1. The representations corresponding to the extremal nodes indicated in (3.6i) correspond to a particle multiplet with highest weight $\mathcal{M}=1 / R_{d}$ (a KK mode), a string multiplet with highest weight $\mathcal{T}_{1}=R_{1} / l_{p}^{3}$ (a singly wound M2-brane), and a membrane multiplet with highest weight $1 / l_{p}^{3}$. The particle and string multiplets are precisely the ones charged under the one- and twoform potentials in Table 1 . The other members in the same multiplet are obtained by applying Weyl reflections on these highest weights, and e.g. for $d=7$, the particle multiplet transforms in the $\mathbf{5 6}$ of $E_{(7(7)}$ with content listed in Table 2. The multiplet consists of the particle states obtained from the KK state and completely wrapped M2, M5 and KK6-branes. It restricts to the lower-dimensional particle multiplets upon decompactification, and decomposes as perturbative states, D-branes and NS-branes under T-duality. We do not display the $d=8$ particle multiplet, but point out that the latter branches into additional representations under T-duality, and in particular contains states with a mass of order $1 / g_{s}^{3}$, akin to the KK6-brane discussed in section 2. This behaviour arises as soon as less than three transverse dimensions are present, and signals a non asymptotically flat geometry.

\section{Borel generators and invariant mass formulae}

Next, we wish to include the Borel generators, corresponding to the right action of nilpotent matrices $N$ in the Iwasawa decomposition (2.3), in order to derive duality invariant mass formulae. In the case of T-duality, the Borel generators include Dehn twists from the mapping class group $S l(d, Z)$, acting on the homology lattice as $\gamma_{I} \rightarrow \gamma_{I}+\gamma_{J}$, as well as the spectral flow of the B-field:

$\Gamma_{i j}: \quad A_{j}^{(i)} \rightarrow A_{j}^{(i)}+1, \quad \Delta_{i j}: \quad B_{i j} \rightarrow B_{i j}+1$

Using the $S l(d, Z)$ boosts, a KK state with momentum 1 can be related to a state with any integer momentum $m_{i}$ along the circles of $T^{d}$ with mass $\mathcal{M}^{2}=m_{i} g^{i j} m_{j}$. More generally, using $(4.1)$, the vector representation of the Weyl group of T-duality becomes a lattice of KK and winding charges $\left(m_{i}, m^{i}\right)$ with mass

$\mathcal{M}_{0}^{2}=\left(m_{i}+B_{i j} m^{j}\right) g^{i k}\left(m_{k}+B_{k l} m^{l}\right)+m^{i} g_{i j} m^{j}$ 


\begin{tabular}{|c|l|l||c|}
\hline mass $\mathcal{M}$ & $S l(7)$ irrep & charge & $E_{\mathrm{YM}}$ \\
\hline$\frac{1}{R_{I}}$ & $\mathbf{7}$ & $m_{1}$ & $\frac{g_{\mathrm{YM}}^{2} s_{I}^{2}}{N V_{s}}$ \\
$\frac{R_{I} R_{J}}{l_{p}^{3}}$ & $\mathbf{2 1}$ & $m^{2}$ & $\frac{V_{s}}{N g_{\mathrm{YM}}^{2}\left(s_{I} s_{J}\right)^{2}}$ \\
$\frac{R_{I} R_{J} R_{K} R_{L} R_{M}}{l_{p}^{6}}$ & $\mathbf{2 1}$ & $m^{5}$ & $\frac{V_{s}^{3}}{N g_{\mathrm{YM}}^{6}\left(s_{I} s_{J} s_{K} s_{L} s_{M}\right)^{2}}$ \\
$\frac{R_{I}^{2} R_{J} R_{K} R_{L} R_{M} R_{N} R_{P}}{l_{p}^{9}}$ & $\mathbf{7}$ & $m^{1 ; 7}$ & $\frac{V_{s}^{5}}{N g_{\mathrm{YM}}^{10}\left(s_{I} ; s_{J} s_{K} s_{L} s_{M} s_{N} s_{P} s_{Q}\right)^{2}}$ \\
\hline
\end{tabular}

Table 2: Particle multiplet, 56 of of $E_{7(7)}$ and Yang-Mills interpretation.

subject to the half-BPS condition $\|m\|^{2}=2 m_{i} m^{i}$ $=0$. For quarter-BPS states the mass formula receives an extra contribution proportional to $\|m\|^{2}$. Similarly, the D-particle states (completely wrapped D-branes) with masses $1 / g_{s} l_{s}, R_{i} R_{j} / g_{s} l_{s}^{3}$, $R_{i} R_{j} R_{k} R_{l} / g_{s} l_{s}^{5}, \ldots$ turn into a lattice of integer D0,D2,D4-charges $m=\left\{m, m^{i j}, m^{i j k l}, \ldots\right\}$ transforming as spinor of $S O(d, d, Z)$. A careful analysis of the Borel generators then gives the half-BPS mass

$$
\mathcal{M}_{0}^{2}=\frac{1}{g_{s}^{2}}\left[\tilde{m}^{2}+\left(\tilde{m}^{i j}\right)^{2}+\left(\tilde{m}^{i j k l}\right)^{2}+\ldots\right]
$$

where the $\tilde{m}=\mathcal{V} \cdot m$ are the dressed charges $\tilde{m}=m+m^{i j} B_{i j}+m^{i j k l} B_{i j} B_{k l}+\ldots, \tilde{m}^{i j}=$ $m^{i j}+m^{k l i j} B_{k l}+\ldots, \tilde{m}^{i j k l}=m^{i j k l}+\ldots$, with an extra contribution when the half-BPS conditions, $k^{i j k l} \equiv m^{[i j} m^{k l]}+m m^{i j k l}=0$ for $d=4$, are not fulfilled. These BPS mass formulae can also be obtained by analyzing the Born-Infeld action $\left[1 \overline{1} \overline{2}_{b}^{\prime}, \overline{6} \bar{b}\right.$.

In the case of the U-duality group, the Borel generators include the usual Dehn twists $\Gamma_{I J}$ in $S l(d, Z)$ as well as the $B_{i j}=\mathcal{C}_{s i j}$ shifts in $S O(d-$ $1, d-1, Z)$, which can be conjugated into $C_{I J K}$ : $\mathcal{C}_{I J K} \rightarrow \mathcal{C}_{I J K}+1$. When $d \geq 6$ we also need to include shifts of the dual gauge fields $\mathcal{E}_{6}, \mathcal{K}_{1 ; 8}$. The U-duality invariant mass formula on tori with arbitrary gauge backgrounds can then again be obtained by considering the non-commuting $\mathcal{C}_{3}, \mathcal{E}_{6}$ and $\mathcal{K}_{1 ; 8}$ flows. In $d=7$, we obtain the result

$\mathcal{M}_{0}^{2}=\left(\tilde{m}_{1}\right)^{2}+\frac{1}{l_{p}^{6}}\left(\tilde{m}^{2}\right)^{2}+\frac{1}{l_{p}^{12}}\left(\tilde{m}^{5}\right)^{2}+\frac{1}{l_{p}^{18}}\left(\tilde{m}^{1 ; 7}\right)^{2}$

where the dressed charges are given by

$$
\begin{aligned}
\tilde{m}_{1}= & m_{1}+\mathcal{C}_{3} m^{2}+\left(\mathcal{C}_{3} \mathcal{C}_{3}+\mathcal{E}_{6}\right) m^{5} \\
& +\left(\mathcal{C}_{3} \mathcal{C}_{3} \mathcal{C}_{3}+\mathcal{C}_{3} \mathcal{E}_{6}\right) m^{1 ; 7} \\
\tilde{m}^{2}= & m^{2}+\mathcal{C}_{3} m^{5}+\left(\mathcal{C}_{3} \mathcal{C}_{3}+\mathcal{E}_{6}\right) m^{1 ; 7} \\
\tilde{m}^{5}= & m^{5}+\mathcal{C}_{3} m^{1 ; 7} \\
\tilde{m}^{1 ; 7}= & m^{1 ; 7}
\end{aligned}
$$

It turns out that the half-BPS condition on the particle multiplet transforms as the string multiplet constructed out of the particle charges [i]1], which also follows on dimensional grounds:

$$
\begin{aligned}
k^{1} & =m_{1} m^{2} \equiv 0 \\
k^{4} & =m_{1} m^{5}+m^{2} m^{2} \equiv 0 \\
k^{1 ; 6} & =m_{1} m^{1 ; 7}+m^{2} m^{5} \equiv 0 \\
k^{3 ; 7} & =m^{2} m^{1 ; 7}+m^{5} m^{5} \equiv 0 \\
k^{6 ; 7} & =m^{5} m^{1 ; 7} \equiv 0
\end{aligned}
$$

When these composite charges do not vanish, the state is at most quarter-BPS, in which case its mass formula reads $\mathcal{M}^{2}=\mathcal{M}_{0}^{2}(m)+\sqrt{[\mathcal{T}(k)]^{2}}$ with $\mathcal{T}(k)$ the half-BPS tension formula for the string multiplet and $k$ the quadratic charges in $(4.6)$.

\section{U-duality in Matrix gauge theory}

Finally, we wish to use the information on Uduality and invariant mass formulae obtained above to obtain a better understanding of the degrees of freedom and symmetries of matrix gauge theory. According to this prescription [110 $\left.0_{1}^{\prime}\right]$ the DLCQ of M-theory on a lightlike circle $S^{1}$ (radius $R_{l}$ ) times $T^{d}$ in the sector with $P_{+}=N / R_{l}$ is described by a supersymmetric $U(N)$ gauge theory in $d+1$ dimensions on the maximal T-dual torus $\tilde{T}^{d}$. In this description the KK state of the particle multiplet bound to $N$ D0-branes becomes (after maximal T-duality) a string wound on $N \mathrm{D} d$ branes with YM energy $E_{\mathrm{YM}}=g_{\mathrm{YM}}^{2} s_{I}^{2} / N V_{s}=$ $\mathcal{M}^{2} / P_{+}$describing a half-BPS state carrying electric flux. On the other hand, a wrapped membrane of the string multiplet bound to $N$ D0branes becomes a $\mathrm{KK}$ state bound to $N \mathrm{D} d$ branes with energy $E_{\mathrm{YM}}=1 / s_{I}=R_{l} \mathcal{T}_{1}$ describing a massless excitation. More generally, the U-duality invariant gauge theory masses can be 
computed as

$$
E_{\mathrm{YM}}=\frac{\mathcal{M}^{2}}{P_{+}}+R_{l} \mathcal{T}_{1}
$$

As an example, the last column of Table 2 gives the corresponding gauge theory masses corresponding to the particle multiplet of $E_{7(7)}$. The first state is the state carrying electric flux mentioned above, while the second state carries magnetic flux, and corresponds in general to a $\mathrm{D} d-\mathrm{D}(d-2)$ bound state. In the case of the string multiplet, the details of which we omit here, the first states are the KK excitations, and the second is a YM instanton in $3+1$ dimensions lifted to $d+1$ dimensions, corresponding to $\mathrm{D} d-\mathrm{D}(d-4)$ bound states. Starting on $T^{5}$, we see the occurrence of states with energy $1 / g_{\text {YM }}^{4}$ whose interpretation is problematic in pure Yang-Mills terms.

We can make a more precise comparison of the U-duality invariant masses with the proposed quantum descriptions for $d=3,4,5$. In the first case the D3-brane description is valid so that we have $\mathrm{N}=4$ supersymmetric gauge theory in $3+1$ dimensions. The U-duality symmetry $S l(3, Z) \times$ $S l(2, Z)$ corresponds to the mapping class group of the three-torus together with the S-duality symmetry of the gauge theory [1 13 in. In particular, the M-theory or type IIB modular parameter $\tau=\mathcal{C}_{123}+i V / l_{p}^{3}$ is equated to $S=\theta / 2 \pi+$ $i 4 \pi / g_{\mathrm{YM}}^{2}$, so that the M-theory gauge potential $\mathcal{C}_{123}$ translates as a $\theta$ angle in the gauge theory. For $d=4$ the quantum theory is the $(2,0)$ worldvolume theory on the M5-brane, where the extra dimension (D4 $\rightarrow$ M5) is generated by identifying $R_{5}=g_{\mathrm{YM}}^{2}$. The $S l(5, Z)$ U-duality symmetry is now naturally interpreted as the symmetry of M5 on the five-torus, and indeed from the invariant masses we can infer directly that $\mathcal{A}^{I}=\epsilon^{I J K L} \mathcal{C}_{J K L}$ appears as the $\mathrm{KK}$ gauge field on $T^{5}=T^{4} \times S^{1}$. Finally, for $d=5$ the quantum theory is the non-critical string theory living on the NS5-brane (obtained by type IIB S-duality from the D5-brane) with string length $\hat{l}_{s}^{2}=g_{\mathrm{YM}}^{2}$. The $S O(5,5, Z)$ U-duality symmetry is now interpreted as the T-duality symmetry of the string theory, and from the invariant masses we infer that $B^{I J}=\epsilon^{I J K L M} \mathcal{C}_{K L M}$ is the $B$-field of string theory on NS5-brane. For $d \geq 6$ gravity does no longer decouple anymore. These conclusions can also be reached from the Born-Infeld action in the scaling limit [1]1]. Finally, we mention that the string and particle multiplet of $E_{d(d)}(Z)$, together with the momentum $N$ and some additional charges, make a string multiplet representation of $E_{d+1(d+1)}(Z)$, as should be the case if

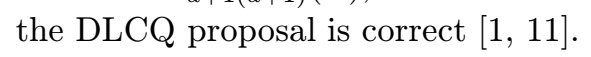

\section{References}

[1] N. A. Obers, B. Pioline, and E. Rabinovici Nucl. Phys. B525 (1998) 163, hep-th/9712084 ; N. A. Obers and B. Pioline hep-th/9809039', to appear in Phys. Rept.

[2] P. K. Townsend Phys. Lett. B350 (1995) 184, hep-th/9501068; E. Witten hep-th/9507121'.

[3] M. B. Green and M. Gutperle Nucl. Phys. B498 (1997) 195, hep-th/9701093.

[4] N. Berkovits thep-th/9709116; B. Pioline Phys. Lett. B431 (1998) 73, hep-th/9804023; M. B. Green and S. Sethi ihep-th/9808061'

[5] E. Kiritsis and B. Pioline Nucl. Phys. $\mathbf{B 5 0 8}$ (1997) 509, hep-th/9707018;

N. A. Obers and B. Pioline, work in progress.

[6] C. Bachas, C. Fabre, E. Kiritsis, N. A. Obers, and P. Vanhove Nucl. Phys. B509 (1998) 33, hep-th/9707126; B. Pioline and E. Kiritsis Phys. Lett. B418 (1998) 61, hep-th/9710078; P. Vanhove, hep-th/9712079; B. Pioline, hep-th/9712155.

[7] C. M. Hull and P. K. Townsend Nucl. Phys. B451 (1995) 525, hep-th/9505073.

[8] E. Cremmer, B. Julia in Superspace and Supergravity, S. W. Hawking and M. Rocek, eds. Cambridge University Press, 1981.

[9] S. Elitzur, A. Giveon, D. Kutasov, and E. Rabinovici_Nucl. Phys. B509 (1998) 122, hep-th/9707217.

[10] T. Banks, W. Fischler, S. H. Shenker, and L. Susskind Phys. Rev. D55 (1997) 5112, hep-th/9610043; L. Susskind hep-th/9704080; A. Sen Adv. Theor. Math. Phys. 2 (1998) 51, 1998, hhep-th/9709220' N. Seiberg Phys. Rev. Lett. $\mathbf{7 9}(1997) 3577$ hep-th/9710009'.

[11] M. Blau and M. O'Loughlin Nucl. Phys. B525 (1998) 182, hep-th/9712047' C. M. Hull $J$. High Energy Phys. $\mathbf{9 8 0 7}$ (1998) 018, hep-th/9712075.

[12] R. Dijkgraaf, E. Verlinde, and H. Verlinde Nucl. Phys. B486 (1997) 89, hep-th/9604055'.

[13] L. Susskind hep-th/9611164; O. J. Ganor, S. Ramgoolam, and W. Taylor IV Nucl. Phys. B492 (1997) 191, hep-th/9611202.. 